\title{
A branching random walk seen from the tip
}

\author{
Éric Brunet · Bernard Derrida
}

October 15,2018

\begin{abstract}
We show that all the time-dependent statistical properties of the rightmost points of a branching Brownian motion can be extracted from the traveling wave solutions of the Fisher-KPP equation. We show that the distribution of all the distances between the rightmost points has a long time limit which can be understood as the delay of the Fisher-KPP traveling waves when the initial condition is modified. The limiting measure exhibits the surprising property of superposability: the statistical properties of the distances between the rightmost points of the union of two realizations of the branching Brownian motion shifted by arbitrary amounts are the same as those of a single realization. We discuss the extension of our results to more general branching random walks.
\end{abstract}

PACS 02.50.-r $\cdot 05.40 .-\mathrm{a} \cdot 89.75 . \mathrm{Hc}$

\section{Introduction}

A branching random walk is a collection of points which, starting from a single point, diffuse and branch independently of the time, of their positions or of the other points, as in figure 1 .
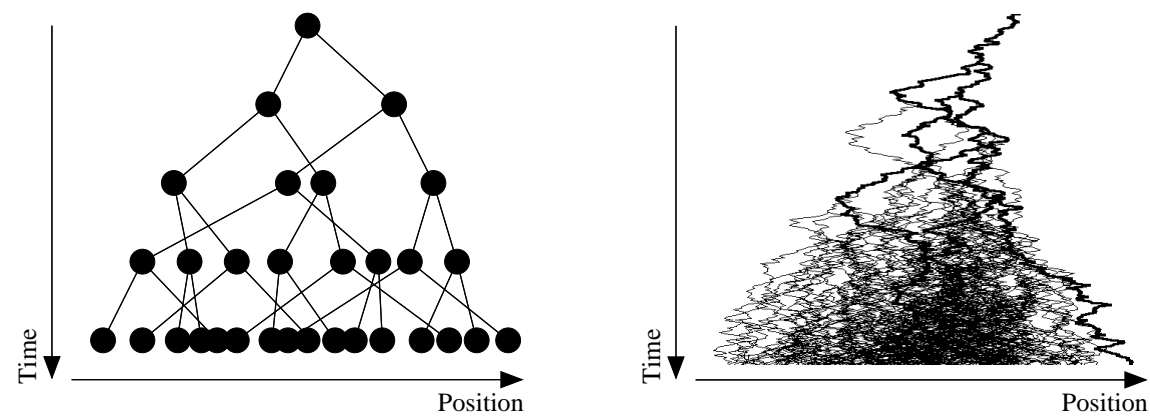

Fig. 1 Two examples of branching random walks. Left: a branching random walk with discrete time where each point splits into two points at each time step. Right: a continuous version called branching Brownian motion where points diffuse as in a Brownian motion and branch with a constant rate.

Laboratoire de Physique Statistique, École Normale Supérieure, UPMC, Université Paris Diderot, CNRS, 24 rue Lhomond, 75005 Paris, France.

E-mail: Eric.Brunet@lps.ens.fr

E-mail: Bernad.Derrida@lps.ens.fr 
Branching random walks appear in many contexts ranging from Mathematics [25,8,22] to Biology [16, 20, 17]. They can for example be used to describe how a growing population invades a new environment. In the one dimensional case, see figure 1, there is, at a given time $t$, a rightmost individual at position $X_{1}(t)$, a second rightmost at $X_{2}(t)$ and so on. (Note that the rightmost $X_{1}\left(t^{\prime}\right)$ at a time $t^{\prime}>t$ is not necessarily a descendant of the rightmost $X_{1}(t)$ at time $t$.) The expected position $m_{t}=\left\langle X_{1}(t)\right\rangle$ of the rightmost individual as well as the probability distribution of its position $X_{1}(t)$ around $m_{t}$ are well understood [8] 25]; the goal of the present paper is to describe the statistical properties of the positions of all the rightmost points in the system, in particular the distribution of the distances between the two rightmost points, the average density of points at some fixed distance from the rightmost $X_{1}(t)$, etc.

One motivation for studying these distances is that the problem belongs to the broader context of extreme value statistics [33,5, 11, 24, 32, 30, 10, 18]: Trying to understand the statistical properties of the rightmost points in a random set of points on the line is a problem common to the studies of the largest eigenvalues of random matrices [33], of the extrema of random signals [12,13, 29,4, 19,6], or of the low lying states of some disordered systems such as spin glasses [26, 30, 7, 1, 2]. In fact, the points generated after some time $t$ by a branching random walk can be viewed as the energies of the configurations of a directed polymer in a random medium [14,27], and the distances between the rightmost points as the gaps between the low lying energy states.

The most studied example of branching random walk is the branching Brownian motion: one starts with a single point at the origin which performs a Brownian motion and branches at a given fixed rate (right part of figure 11. Whenever a branching event occurs, the point is replaced by two new points which evolve themselves as two independent branching Brownian motions. While the number of points generated after some time $t$ grows exponentially with time, the expected position $m_{t}$ of the rightmost point increases only linearly with time [25],8]. In one dimension, Mc Kean [25] and Bramson [8] have shown that the probability distribution of the rightmost point is given by the traveling wave solution of the Fisher-KPP equation, with a step initial condition. Here we will see that all the statistical properties of the rightmost points can be understood in terms of solutions to the Fisher-KPP equation with appropriate initial conditions [9]. We will also show that the distribution of the distances between these rightmost points has a long time limit which exhibits the striking property of superposability: the distances between the rightmost points of the union of two realizations of the branching Brownian motion have the same statistics as those of a single realization.

This paper is organized as follows: in section 2 we introduce some generating functions useful to study random sets of points on the line and show how one can use them to obtain all the properties of these random sets. In section 3 we show that, for the branching Brownian motion, all these generating functions are solutions of the Fisher-KPP equation. We also show that the distribution of all the rightmost points as seen from $m_{t}$ or, alternatively, as seen from $X_{1}(t)$, has a long time limit which can be computed as the delay of Fisher-KPP traveling waves. This distribution has the property of superposability. In section 4. we present results, mostly numerical, on some specific aspects of the limiting distribution of points in the branching Brownian motion, namely the distribution of the distance between the two rightmost points and the average density seen from the rightmost point. In section 5 we explain how the results on the branching Brownian motion can be extended to more general branching random walks. Finally, we study in section 6 the distribution of all the rightmost points in a specific frame which depends on the realization and which was introduced by Lalley and Sellke [22].

\section{Statistics of point measures on the line}

In this section, we introduce some useful quantities (generating functions) to characterize random sets of points on the line such that the number $n(x)$ defined as

$$
n(x)=\text { (the number of points on the right of position } x \text { ) }
$$


is finite and vanishes for $x$ large enough.

2.1 The generating functions

The first generating function one can define is

$$
\psi_{\lambda}(x)=\left\langle\lambda^{n(x)}\right\rangle .
$$

From the knowledge of this function, one can extract the probability distribution function $p_{i}(x)$ of the position $x$ of the $i$-th rightmost point. Indeed, by definition (2) of $\psi_{\lambda}$,

$$
\psi_{\lambda}(x)=\sum_{i \geq 0} Q_{i}(x) \lambda^{i},
$$

where $Q_{i}(x)$ is the probability that there are exactly $i$ points on the right of $x$. One can notice that $Q_{0}(x)+$ $Q_{1}(x)+\cdots+Q_{i-1}(x)$ is the probability to have less than $i$ points on the right of $x$. The generating function of these sums is, from (3),

$$
\frac{\lambda}{1-\lambda} \psi_{\lambda}(x)=Q_{0}(x) \lambda+\left[Q_{0}(x)+Q_{1}(x)\right] \lambda^{2}+\left[Q_{0}(x)+Q_{1}(x)+Q_{2}(x)\right] \lambda^{3}+\cdots .
$$

But $Q_{0}(x)+Q_{1}(x)+\cdots+Q_{i-1}(x)$ is also the probability that the $i$-th rightmost point, if it exists, is on the left of $x$. Therefore,

$$
\frac{\lambda}{1-\lambda} \partial_{x} \psi_{\lambda}(x)=\sum_{i \geq 1} p_{i}(x) \lambda^{i},
$$

where $p_{i}(x) \mathrm{d} x$ is the probability that the $i$-th rightmost point exists and is in the interval $[x, x+\mathrm{d} x]$. (Note that $\int p_{i}(x) \mathrm{d} x \leq 1$ is the probability that there are at least $i$ points on the line.)

The knowledge of $\psi_{\lambda}(x)$ gives in particular the average distances between the points: from (5), one can see that

$$
\begin{aligned}
\int \mathrm{d} x x \partial_{x} \psi_{\lambda}(x) & =(1-\lambda)\left[\left\langle X_{1}\right\rangle+\lambda\left\langle X_{2}\right\rangle+\lambda^{2}\left\langle X_{3}\right\rangle+\cdots\right], \\
& =\left\langle X_{1}\right\rangle-\lambda\left[\left\langle X_{1}\right\rangle-\left\langle X_{2}\right\rangle\right]-\lambda^{2}\left[\left\langle X_{2}\right\rangle-\left\langle X_{3}\right\rangle\right]-\cdots,
\end{aligned}
$$

where $\left\langle X_{i}\right\rangle=\int x p_{i}(x) \mathrm{d} x$ is the average position of the $i$-th point (with the convention that $X_{i}=0$ if there are less than $i$ points in the system). Therefore

$$
\int \mathrm{d} x x\left[\partial_{x} \psi_{0}(x)-\partial_{x} \psi_{\lambda}(x)\right]=\sum_{i \geq 1}\left\langle d_{i, i+1}\right\rangle \lambda^{i}
$$

where $\left\langle d_{i, i+1}\right\rangle=\left\langle X_{i}\right\rangle-\left\langle X_{i+1}\right\rangle$ is the average distance between the $i$-th and the $(i+1)$-th point.

To obtain the correlations between the positions of pairs of points, one can start, for $y<x$, from the generating function

$$
\psi_{\lambda \mu}(x, y)=\left\langle\lambda^{n(x)} \mu^{n(y)}\right\rangle .
$$

The coefficient in front of $\lambda^{i} \mu^{j}$ in the expansion of $\psi_{\lambda \mu}$ in powers of $\lambda$ and $\mu$ is the probability that there are exactly $i$ points on the right of $x$ and $j$ points on the right of $y$. As in (4), the coefficient of $\lambda^{i} \mu^{j}$ in the expansion of $\lambda /(1-\lambda) \times \mu /(1-\mu) \times \psi_{\lambda \mu}(x, y)$ is the probability that there are less than $i$ points on the right of $x$ and less than $j$ points on the right of $y$, which is also the probability that the $i$-th rightmost point (if it exists) is on the left of $x$ and the $j$-th rightmost point (if it exists) is on the left of $y$. Thus, for $y<x$,

$$
\frac{\lambda}{1-\lambda} \frac{\mu}{1-\mu} \partial_{x} \partial_{y} \psi_{\lambda \mu}(x, y)=\sum_{\substack{i>1 \\ j>i}} p_{i j}(x, y) \lambda^{i} \mu^{j},
$$


where $p_{i j}(x, y) \mathrm{d} x \mathrm{~d} y$ is the probability that both the $i$-th and $j$-th rightmost points exist and lie respectively in the intervals $[x, x+\mathrm{d} x]$ and $[y, y+\mathrm{d} y]$.

One can generalize (27) by defining, for $x_{0}>x_{1}>\cdots>x_{k}$, the generating functions

$$
\psi_{\lambda_{0}, \ldots, \lambda_{k}}\left(x_{0}, \ldots, x_{k}\right)=\left\langle\lambda_{0}^{n\left(x_{0}\right)} \cdots \lambda_{k}^{n\left(x_{k}\right)}\right\rangle
$$

of the numbers $n\left(x_{0}\right), \ldots, n\left(x_{k}\right)$ of points on the right of positions $x_{0}, \ldots, x_{k}$, and get as in 58 all the higher correlation functions. In that way, all the statistical properties of the measure can be derived from the knowledge of the generating functions 9 ).

\subsection{The measure seen from the rightmost point}

In the following we will often try to characterize the random set of points as seen from the rightmost point (i.e. in the frame where the rightmost point is at the origin). To do so, let us define the generating functions of the numbers $m(z)$ of points at the right of $z$ in the frame of the rightmost point. (Note that if $X_{1}$ is the position of the rightmost, then $m(z)=n\left(X_{1}+z\right)$ and one has $m(z) \geq 1$ for $z<0$ and $m(z)=0$ for $z>0$.)

$$
\chi_{\lambda_{1}, \ldots, \lambda_{k}}\left(z_{1}, \ldots, z_{k}\right)=\left\langle\lambda_{1}^{m\left(z_{1}\right)} \cdots \lambda_{k}^{m\left(z_{k}\right)}\right\rangle
$$

(As in (9), we assume $z_{1}>z_{2}>\cdots>z_{k}$.) These generating functions, as in section 2.1, allow one to calculate all the statistical properties of the measure in the frame of the rightmost point (in particular the distribution of the relative distances between the points). They can be determined from the knowledge of the generating functions $\psi_{\lambda_{0}, \ldots, \lambda_{k}}\left(x_{0}, \ldots, x_{k}\right)$ defined in 9 by

$$
\chi_{\lambda_{1}, \ldots, \lambda_{k}}\left(z_{1}, \ldots, z_{k}\right)=\int \mathrm{d} x \partial_{x_{0}} \psi_{0, \lambda_{1}, \ldots, \lambda_{k}}\left(x, x+z_{1}, \ldots, x+z_{k}\right)
$$

In section 4 we will calculate the density of probability $P_{12}(a)$ that the two rightmost points are separated by a distance $a$ (and that there are at least two points on the line) and the average density $\rho(a)$ at a distance $a$ from the rightmost point. From 10 , one can see that

$$
P_{12}(a)=-\left.\partial_{a} \partial_{\mu} \chi_{\mu}(-a)\right|_{\mu=0}=-\left.\partial_{a} \int \mathrm{d} x \partial_{\mu} \partial_{x_{0}} \psi_{0 \mu}(x, x-a)\right|_{\mu=0}
$$

Then using that $\partial_{x_{0}} \psi_{0 \mu}(x, x-a)=\left(\partial_{x}+\partial_{a}\right) \psi_{0 \mu}(x, x-a)$, one gets

$$
P_{12}(a)=-\left.\partial_{a}^{2} \int \mathrm{d} x \partial_{\mu} \psi_{0 \mu}(x, x-a)\right|_{\mu=0}
$$

By a similar calculation one can show that the average density $\rho(a)$ of points at distance $a$ from the rightmost point is

$$
\rho(a)=\left.\partial_{a}^{2} \int \mathrm{d} x \partial_{\mu} \psi_{0 \mu}(x, x-a)\right|_{\mu=1}
$$

\subsection{Examples}

We now describe a few examples of such measures. 


\subsubsection{A Poisson process with an arbitrary density $r(x)$}

Our first example is a Poisson process on the line with a density $r(x)$. We assume that $r(x)$ decays fast enough to the right so that a rightmost point exists, and that $\int r(x) \mathrm{d} x=\infty$ so that there are infinitely many points on the line.

By definition of a Poisson process, each infinitesimal interval $[x, x+\mathrm{d} x]$ is occupied by a point with probability $r(x) \mathrm{d} x$ and empty with probability $1-r(x) \mathrm{d} x$, and the occupation numbers of disjoint intervals are uncorrelated. The probability $Q_{i}(x)$ that there are exactly $i$ points on the right of $x$ is given by

$$
Q_{i}(x)=\frac{R(x)^{i} e^{-R(x)}}{i !} \quad \text { where } \quad R(x)=\int_{x}^{\infty} r(z) \mathrm{d} z .
$$

From this, we obtain $\psi_{\lambda}(x)$ from $\sqrt{23}$ and $\psi_{\lambda \mu}(x, y)$ from 77 in the Poisson process:

$$
\psi_{\lambda}(x)=e^{-(1-\lambda) R(x)}, \quad \psi_{\lambda \mu}(x, y)=e^{-\mu(1-\lambda) R(x)-(1-\mu) R(y)} .
$$

Using (6), the generating function of the average $\left\langle d_{i, i+1}\right\rangle$ between the $i$-th and $(i+1)$-th points is

$$
\sum_{i \geq 1} \lambda^{i}\left\langle d_{i, i+1}\right\rangle=\int_{-\infty}^{\infty} \mathrm{d} x\left[e^{-(1-\lambda) R(x)}-e^{-R(x)}\right]
$$

The probability distribution function $P_{12}(a)$ that the distance $d_{1,2}$ is equal to $a$ and the average density $\rho(a)$ seen at a distance $a$ from the rightmost point are given by

$$
P_{12}(a)=\int_{-\infty}^{\infty} \mathrm{d} x r(x+a) r(x) e^{-R(x)}, \quad \rho(a)=\int_{-\infty}^{\infty} \mathrm{d} x r(x-a) r(x) e^{-R(x)} .
$$

These expressions can be understood directly from the definition of the Poisson process or, with a little more algebra, from 13 14 . One can notice that $P_{12}(a)$ and $\rho(a)$ are given by the same expression with $a$ replaced by $-a$ and are therefore analytic continuations of each other whenever $r(x)$ is analytic.

\subsubsection{A Poisson process with an exponential density $e^{-\alpha x}$}

In the special case where the density of the Poisson process is an exponential $r(x)=\exp (-\alpha x)$, one can simply replace $R(x)$ in the previous expressions by $\exp (-\alpha x) / \alpha$. This gives

$$
\begin{aligned}
\psi_{\lambda}(x) & =\exp \left[-(1-\lambda) \frac{e^{-\alpha x}}{\alpha}\right]=\exp \left[-e^{-\alpha\left(x-\frac{\ln (1-\lambda)}{\alpha}\right)}\right], \\
\psi_{\lambda \mu}(x, y) & =\exp \left[-\mu(1-\lambda) \frac{e^{-\alpha x}}{\alpha}-(1-\mu) \frac{e^{-\alpha y}}{\alpha}\right],
\end{aligned}
$$

so that from 6

$$
\sum_{i \geq 1} \lambda^{i}\left\langle d_{i, i+1}\right\rangle=-\frac{\ln (1-\lambda)}{\alpha}
$$

and thus 9

$$
\left\langle d_{i, i+1}\right\rangle=\frac{1}{\alpha i}
$$

One also has from 18

$$
P_{12}(a)=\alpha e^{-\alpha a}, \quad \rho(a)=\alpha e^{\alpha a}
$$




\subsubsection{Decorated measures}

Start with a collection of points $u_{i}$, distributed according to some measure $v_{1}$ and, independently for each point $u_{i}$, replace it by a realization of another measure $v_{2}$ shifted by $u_{i}$. We say that the points $u_{i}$ are decorated by the measure $v_{2}$ and call the resulting measure as $v_{1}$ decorated by $v_{2}$.

We assume that $v_{1}$ and $v_{2}$ are such that the decorated measure has a rightmost point. If the functions $\psi_{\lambda}(x), \psi_{\lambda \mu}(x, y), \ldots$ for the measure $v_{2}$ are known, the decorated measure is characterized by functions $\Psi_{\lambda}(x), \Psi_{\lambda \mu}(x, y), \ldots$ given by

$$
\Psi_{\lambda}(x)=\left\langle\prod_{i} \psi_{\lambda}\left(x-u_{i}\right)\right\rangle_{u_{i}}, \quad \Psi_{\lambda \mu}(x, y)=\left\langle\prod_{i} \psi_{\lambda \mu}\left(x-u_{i}, y-u_{i}\right)\right\rangle_{u_{i}},
$$

where the average is over all realizations $\left\{u_{i}\right\}$ of the measure $v_{1}$. For instance, if $v_{1}$ is a Poisson process of density $r(u)$, then

$$
\begin{gathered}
\Psi_{\lambda}(x)=\prod_{u}\left[1-r(u) \mathrm{d} u+r(u) \psi_{\lambda}(x-u) \mathrm{d} u\right]=\exp \left[\int\left[\psi_{\lambda}(x-u)-1\right] r(u) \mathrm{d} u\right] \\
\Psi_{\lambda \mu}(x, y)=\exp \left[\int\left[\psi_{\lambda \mu}(x-u, y-u)-1\right] r(u) \mathrm{d} u\right]
\end{gathered}
$$

\subsubsection{Ruelle cascades}

For a decorated measure where the decoration $v_{2}$ is a Poisson process of density $e^{-\alpha x}$, the average over the $u_{i}$ 's in 23. leads in general to complicated expressions for $\Psi_{\lambda}(x)$ or $\Psi_{\lambda \mu}(x, y)$. The expressions for $P_{12}(a)$ and $\rho(a)$ are however the same as in 22) for the pure Poisson process of density $e^{-\alpha x}$. In fact, all the statistical properties of the distances between the rightmost points are the same as those in the exponential Poisson process.

This can be understood from the following reason: decorating the points $u_{1}, \ldots, u_{k}, \ldots$ by independent realizations of a Poisson process of density $e^{-\alpha x}$ is equivalent to drawing a single realization of a Poisson process of density $\sum_{k} e^{-\alpha\left(x-u_{k}\right)}=e^{-\alpha x} \sum_{k} e^{\alpha u_{k}}$, which is just the same as one realization of a Poisson process of density $e^{-\alpha x}$ shifted by the random variable $\ln \left(\sum_{k} e^{\alpha u_{k}}\right) / \alpha$.

The same argument applies to Ruelle cascades, which can be defined as follows $[29,6,7,4]$ : take an increasing sequence of positive numbers $\alpha_{1}<\alpha_{2}<\cdots$ and start with a Poisson process of density $e^{-\alpha_{1} x}$. At each step $k>1$, each point in the system is decorated by a Poisson process of density $e^{-\alpha_{k} x}$. At step $k$, the measure of points in the system is simply, from the previous argument, a Poisson process of density $e^{-\alpha_{k} x}$ globally shifted by a random variable which depends on the positions of the points at step $k-1$. Therefore, the statistics of the distances of the rightmost points is the same as for the Poisson process of density $e^{-\alpha_{k} x}$.

\section{The branching Brownian motion and Fisher-KPP fronts}

\subsection{The Fisher-KPP equation}

We are now going to see how the generating functions (2/7) 9 can be determined when the random set of points on the line are the points generated at time $t$ by a branching Brownian motion.

To define the branching Brownian motion we start at time $t=0$ with a single point at the origin. This point diffuses and branches, and its offspring do the same. After some time $t$, a realization of the process consists of a finite number of points located at positions $X_{i}(t)$ for $i=1,2,3, \ldots$ Then, during the next time interval $\mathrm{d} t \ll 1$, each point, independently of what the others do, moves a random distance 
$X_{i}(t+\mathrm{d} t)-X_{i}(t)=\eta_{i}(t) \sqrt{2 \mathrm{~d} t}$ with $\left\langle\eta_{i}(t)\right\rangle=0$ and $\left\langle\eta_{i}(t)^{2}\right\rangle=1$, and, with probability $\mathrm{d} t$, is replaced by two new points located at the same position $X_{i}(t)$.

For any function $\phi$ one can define the generating function $H_{\phi}(x, t)$ by

$$
H_{\phi}(x, t)=\left\langle\prod_{i} \phi\left[x-X_{i}(t)\right]\right\rangle,
$$

where the $X_{i}(t)$ for $i=1, \ldots, N_{t}$ are the positions of the $N_{t}$ points of the branching Brownian motion at time $t$ and $\langle\cdot\rangle$ denotes an average over all the possible realizations.

By analyzing what happens during the very first time interval $\mathrm{d} t$, one can see that the evolution of $H_{\phi}(x, t)$ satisfies

$$
H_{\phi}(x, t+\mathrm{d} t)=(1-\mathrm{d} t)\left\langle H_{\phi}(x-\eta \sqrt{2 \mathrm{~d} t}, t)\right\rangle_{\eta}+\mathrm{d} t H_{\phi}(x, t)^{2} .
$$

The first term in the right hand side represents the motion of the initial point during the first time interval $\mathrm{d} t$ and the second term represents the branching event which occurs with probability $\mathrm{d} t$ during this first time interval. Taking $\mathrm{d} t$ to zero, one gets

$$
\partial_{t} H_{\phi}=\partial_{x}^{2} H_{\phi}+H_{\phi}^{2}-H_{\phi},
$$

which is the Fisher-KPP equation [16,21,25]. (The Fisher-KPP equation is often written as $\partial_{t} h=\partial_{x}^{2} h+$ $h-h^{2}$, but this is identical to 27) by the change of variable $h=1-H_{\phi}$.) Because there is a single point at the origin at time $t=0$, the initial condition is simply, from [25],

$$
H_{\phi}(x, 0)=\phi(x) .
$$

The generating function $(9)$ at time $t$

$$
\psi_{\lambda_{0}, \ldots, \lambda_{k}}\left(x_{0}, \ldots, x_{k}\right)=\left\langle\lambda_{0}^{n\left(x_{0}\right)} \cdots \lambda_{k}^{n\left(x_{k}\right)}\right\rangle
$$

can be written, for $0>z_{1}>\cdots>z_{k}$, as

$$
\psi_{\lambda_{0}, \ldots, \lambda_{k}}\left(x, x+z_{1}, \ldots, x+z_{k}\right)=\left\langle\prod_{i} \phi\left[x-X_{i}(t)\right]\right\rangle=H_{\phi}(x, t),
$$

where the function $\phi(x)$ is given by

$$
\phi(x)=\lambda_{0}^{1-\theta(x)} \lambda_{1}^{1-\theta\left(x+z_{1}\right)} \cdots \lambda_{k}^{1-\theta\left(x+z_{k}\right)},
$$

and where $\theta(x)$ is the Heaviside step function defined by

$$
\theta(x)= \begin{cases}1 & \text { for } x \geq 0 \\ 0 & \text { for } x<0\end{cases}
$$

See figure 2 for the general shape of 31.

With the choice (31) of $\phi$, the generating function (9) and, therefore, all the properties of the point measure in the branching Brownian motion at time $t$ can be obtained as solutions of the Fisher-KPP equation with the initial condition (28).

In the special case $k=0$ and $\lambda_{0}=0$ of (31), i.e. for the initial condition $\phi(x)=\theta(x)$, one gets

$$
H_{\theta}(x, t)=\operatorname{Proba}(\text { There are no point at time } t \text { on the right of } x),
$$




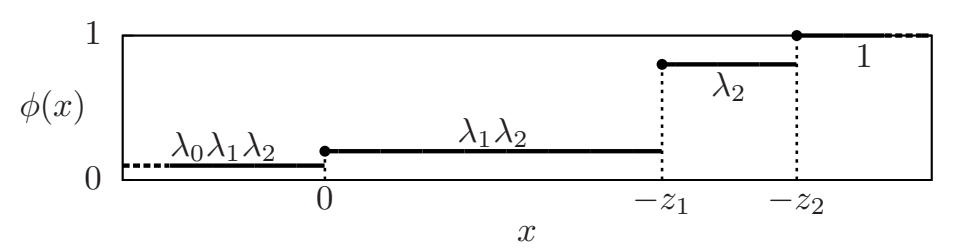

Fig. 2 The function 31 for $k=2$.

and one recovers the well-known fact $[25,8]$ that the solution $H_{\theta}(x, t)$ of the Fisher-KPP equation with a step initial condition is the cumulative distribution function of the position of the rightmost point.

In section 4 we will choose $\phi=\phi_{1}$ and $\phi=\phi_{2}$, other special cases of (31), given by

$$
\phi_{1}(x)=\left\{\begin{array}{ll}
1 & \text { for } x \geq 0, \\
\lambda & \text { for } x<0,
\end{array} \quad \phi_{2}(x)= \begin{cases}1 & \text { for } x \geq a, \\
\mu & \text { for } 0 \leq x<a, \\
\lambda \mu & \text { for } x<0,\end{cases}\right.
$$

to calculate the generating functions $(2,7)$ at time $t$

$$
\psi_{\lambda}(x)=H_{\phi_{1}}(x, t), \quad \psi_{\lambda \mu}(x, x-a)=H_{\phi_{2}}(x, t)
$$

needed to determine the distribution $P_{12}(a)$ and the density $\rho(a)$ defined at the end of section 2

\subsection{The branching Brownian motion seen from the rightmost point}

The Fisher-KPP equation 27] has two homogeneous solutions: $H_{\phi}=1$, which is unstable, and $H_{\phi}=0$, which is stable. When the initial condition $\phi(x)$ is given by the step function $\theta(x)$, see (32), the solution $H_{\theta}(x, t)$ of 27) becomes a traveling wave with the phase $H_{\theta}=0$ invading the phase $H_{\theta}=1$ [16, 21, 8]. As the front is an extended object, one can define its position $m_{t}$ in several ways; for example one could define $m_{t}$ as the solution of $H_{\theta}\left(m_{t}, t\right)=\alpha$ for some $0<\alpha<1$. Here it will be convenient to use the following definition

$$
m_{t}=\int \mathrm{d} x x \partial_{x} H_{\theta}(x, t) .
$$

One can see using (33) that $m_{t}$ defined by 36 ) is the average position of the rightmost point.

If the initial condition 28 is not a step function but is such that $\phi(x)=1$ for all large enough $x$ and $\phi(x)$ is a constant smaller than 1 for all large negative $x$, as in 3134$)$, the solution $H_{\phi}(x, t)$ of 27 ) becomes also a traveling wave. Its position $m_{t}^{(\phi)}$ can be defined as in 36 by

$$
m_{t}^{(\phi)}=\int \mathrm{d} x x \partial_{x} H_{\phi}(x, t)
$$

We are now going to show that the whole measure seen from the rightmost point can be written in terms of this position $m_{t}^{(\phi)}$ : one can rewrite 11 as

$$
\begin{aligned}
\chi_{\lambda_{1}, \ldots, \lambda_{k}}\left(z_{1}, \ldots, z_{k}\right)= & \int \mathrm{d} x\left(\partial_{x}-\partial_{z_{1}}-\cdots-\partial_{z_{k}}\right) \psi_{0, \lambda_{1}, \ldots, \lambda_{k}}\left(x, x+z_{1}, \ldots, x+z_{k}\right), \\
= & \left.\psi_{0, \lambda_{1}, \ldots, \lambda_{k}}\left(x, x+z_{1}, \ldots, x+z_{k}\right)\right|_{x=-\infty} ^{x=+\infty} \\
& -\int \mathrm{d} x\left(\partial_{z_{1}}+\cdots+\partial_{z_{k}}\right) \psi_{0, \lambda_{1}, \ldots, \lambda_{k}}\left(x, x+z_{1}, \ldots, x+z_{k}\right), \\
= & 1+\left(\partial_{z_{1}}+\cdots+\partial_{z_{k}}\right) \int \mathrm{d} x x \partial_{x} \psi_{0, \lambda_{1}, \ldots, \lambda_{k}}\left(x, x+z_{1}, \ldots, x+z_{k}\right) .
\end{aligned}
$$


Then from (30) and 37) one gets

$$
\chi_{\lambda_{1}, \ldots, \lambda_{k}}\left(z_{1}, \ldots, z_{k}\right)=1+\left(\partial_{z_{1}}+\cdots+\partial_{z_{k}}\right) m_{t}^{(\phi)},
$$

where $\phi$ is the function $(31)$ with $\lambda_{0}=0$.

Therefore, with the definition (37) of the position of the front, the whole information about the measure in the frame of the rightmost point, at any time $t$, can be extracted from the $\phi$ dependence of $m_{t}^{(\phi)}$.

3.3 The limiting measure and the delays

In the long time limit, it is known [8,31] that the traveling wave solution $H_{\theta}(x, t)$ of $[27$, , with the initial condition 32, takes an asymptotic shape, $F(x)$. This means that

$$
H_{\theta}\left(m_{t}+x, t\right) \underset{t \rightarrow \infty}{\longrightarrow} F(x),
$$

where $F(x)$ satisfies

$$
F^{\prime \prime}+2 F^{\prime}+F^{2}-F=0, \quad F(-\infty)=0, \quad F(\infty)=1, \quad \int \mathrm{d} x x \partial_{x} F(x)=0 .
$$

It is also known, since the work of Bramson [8], that, in the long time limit, the traveling wave moves at a velocity 2 and that its position (36) is given by

$$
m_{t}=2 t-\frac{3}{2} \ln t+\text { Constant }+o(1) .
$$

If the function $\phi(x)$ is not the step function but is of the form 31 34, the solution $H_{\phi}(x, t)$ of 27p becomes also a traveling wave with the same shape $F(x)$. This wave is centered around the position $m_{t}^{(\phi)}$, defined in [37), and one has

$$
H_{\phi}\left(m_{t}^{(\phi)}+x, t\right) \underset{t \rightarrow \infty}{\longrightarrow} F(x) .
$$

For large times $m_{t}^{(\phi)}$ is still given by $[42$, but with a different constant [8]. This means that

$$
m_{t}-m_{t}^{(\phi)} \underset{t \rightarrow \infty}{\longrightarrow} f[\phi],
$$

where $f[\phi]$ is the long time delay in the position of the front due to the modified initial condition, as compared to a front starting with a step function. Taken together, 433) and 444 give

$$
H_{\phi}\left(m_{t}+x, t\right) \underset{t \rightarrow \infty}{\longrightarrow} F(x+f[\phi]) .
$$

Using (30), this becomes

$$
\psi_{\lambda_{0}, \ldots, \lambda_{k}}\left(m_{t}+x, m_{t}+x+z_{1}, \ldots, m_{t}+x+z_{k}\right) \underset{t \rightarrow \infty}{\longrightarrow} F(x+f[\phi]),
$$

which shows that the measure of $\left\{X_{1}(t)-m_{t}, X_{2}(t)-m_{t}, \ldots\right\}$ (the rightmost points in the branching Brownian motion seen from the $m_{t}$ frame) does converge when $t \rightarrow \infty$ to a limiting point measure characterized by the functions $F(x)$ and $f[\phi]$.

The measure of $\left\{X_{2}(t)-X_{1}(t), X_{3}(t)-X_{1}(t), \ldots\right\}$ (the rightmost points in the branching Brownian motion seen from the $X_{1}(t)$ frame) also has a well-defined limit when $t \rightarrow \infty$. Indeed, using (39) and (44), one gets

$$
\chi_{\lambda_{1}, \ldots, \lambda_{k}}\left(z_{1}, \ldots, z_{k}\right) \underset{t \rightarrow \infty}{\longrightarrow} 1-\left(\partial_{z_{1}}+\cdots+\partial_{z_{k}}\right) f[\phi] .
$$

Therefore, in the long time limit, all the information on the distribution of the rightmost points seen from $X_{1}(t)$ is contained in the $\phi$ dependence of the delay $f[\phi]$.

Note that, in contrast to 39$]$ which requires the position to be defined by $[37]$, the delay $f[\phi]$ in 446 or 477 depends only on $\phi$ : it would not change if we had chosen another definition of the front position. 


\subsection{The superposability of branching Brownian motions}

Let us now consider $M$ independent branching Brownian motions starting at $t=0$ at positions $u_{1}, \ldots, u_{M}$. Following the same argument as in section 3.1, the generating function (9) of the union of the points at time $t$ of these $M$ branching Brownian motions is given by the following generalization of (30)

$$
\psi_{\lambda_{0}, \ldots, \lambda_{k}}\left(x, x+z_{1}, \ldots, x+z_{k}\right)=\prod_{\alpha=1}^{M} H_{\phi}\left(x-u_{\alpha}, t\right),
$$

where $H_{\phi}(x, t)$ is the same solution of 27 with the initial condition $\sqrt{31}$ as in the case of a single branching Brownian motion starting at the origin. In the long time limit, using (45),

$$
\psi_{\lambda_{0}, \ldots, \lambda_{k}}\left(m_{t}+x, m_{t}+x+z_{1}, \ldots, m_{t}+x+z_{k}\right) \underset{t \rightarrow \infty}{\longrightarrow} \prod_{\alpha=1}^{M} F\left(x+f[\phi]-u_{\alpha}\right)
$$

This means that here again, there is a limiting measure when $t \rightarrow \infty$ for the rightmost points in the $m_{t}$ frame. This measure is not the same as before (when one starts with a single point at the origin), as can be seen by comparing (49) and (46). In particular, the distribution of the rightmost point is different.

In the frame of the rightmost point, however, one can see using 49] and (38) that

$$
\chi_{\lambda_{1}, \ldots, \lambda_{k}}\left(z_{1}, \ldots, z_{k}\right) \underset{t \rightarrow \infty}{\longrightarrow} 1-\left(\partial_{z_{1}}+\cdots+\partial_{z_{k}}\right) f[\phi]
$$

as in (47).

It is remarkable that the generating function $\chi$ does depend neither on the number $M$ of starting points nor on their positions $u_{\alpha}$. The picture which emerges is that if we superpose the rightmost points of several branching Brownian motions, starting at arbitrary positions, the limiting measure in the frame of the rightmost point is, when $t \rightarrow \infty$, the same as for a single branching Brownian motion.

We will say that, in the long time limit, the measure of the distances between the rightmost points in a branching Brownian motion becomes superposable: the union of two (or more) realizations of the process (even moved by arbitrary translations $u_{\alpha}$ ) leads to the same measure in the frame of the rightmost point as for a single branching Brownian motion.

As a remark, it is easy to check, that the Poisson process with an exponential density $r(x)=e^{-\alpha x}$, see section 2.3.2 is an example of a superposable measure: the superposition of $M$ such Poisson processes translated by arbitrary amounts $u_{1}, \ldots, u_{M}$ is identical to a single Poisson process with an exponential distribution translated by $\alpha^{-1} \ln \left(e^{\alpha u_{1}}+\cdots+e^{\alpha u_{M}}\right)$. One can also check that, for the same reason, all the decorated measures of section 2.3.3 are superposable when $v_{1}$ is a Poisson process with an exponential density.

In section 6, we will state a stronger version of the superposability property of the branching Brownian motion.

\section{Some quantitative properties of the branching Brownian motion seen from the rightmost point}

In this section we obtain, by integrating numerically the equation 27 with the appropriate initial condition, some statistical properties of the limiting measure seen from the rightmost point. 
4.1 Average distances between consecutive points

The analytic calculation of the delay $f[\phi]$ is in general not easy. For $\phi=\phi_{1}$ given by (34), however, it was possible to show [9] that when $1-\lambda \ll 1$, the delay is given by

$$
f\left[\phi_{1}\right] \simeq-\ln (1-\lambda)-\ln [-\ln (1-\lambda)]+\mathscr{O}(1),
$$

and, from this, one could deduce that, in the long time limit, the average of the distance $d_{i, i+1}$ between the $i$-th and the $(i+1)$-th rightmost points is given for large $i$ by

$$
\left\langle d_{i, i+1}\right\rangle \simeq \frac{1}{i}-\frac{1}{i \ln i}
$$

In [9], the numerical values of the distances between the rightmost points were also obtained by integrating the Fisher-KPP equation with the initial condition $\phi_{1}$ in (34) and by using (6) (in practice we integrated numerically the equations satisfied by the coefficients of the expansion of $\psi_{\lambda}(x)$ in powers of $\lambda)$. It was found that

$$
\begin{array}{ll}
\left\langle d_{1,2}\right\rangle \simeq 0.496, \quad\left\langle d_{2,3}\right\rangle \simeq 0.303, & \left\langle d_{3,4}\right\rangle \simeq 0.219, \\
\left\langle d_{4,5}\right\rangle \simeq 0.172, & \left\langle d_{5,6}\right\rangle \simeq 0.142, \quad\left\langle d_{6,7}\right\rangle \simeq 0.121 .
\end{array}
$$

The results 5253) gave evidence that the distances between the rightmost points of the branching Brownian motion were different from those of a Poisson process with an exponential density [21].

\subsection{Distribution of the distance between the two rightmost points}

According to 13$]$, to obtain the distribution $P_{12}(a)$ of the distance between the two rightmost points, one needs to calculate $\psi_{0 \mu}(x, x-a)$ to first order in $\mu$. We first remark that at time $t$ for $a>0, \psi_{00}(x, x-a)=$ $\operatorname{Proba}[n(x)=0$ and $n(x-a)=0]=\operatorname{Proba}[n(x-a)=0]=H_{\theta}(x-a, t)$ where $H_{\theta}(x, t)$ is the standard Fisher-KPP front with the step initial condition (it is also easy to see from the definition (34) of $\phi_{2}$.) Then writing at time $t$ that

$$
\psi_{0 \mu}(x, x-a)=H_{\theta}(x-a, t)+\mu R_{a}(x-a, t)+\mathscr{O}\left(\mu^{2}\right)
$$

is solution of the Fisher-KPP equation, and using the initial condition $\phi_{2}$ in (34), one gets

$$
\partial_{t} R_{a}=\partial_{x}^{2} R_{a}-R_{a}+2 H_{\theta} R_{a} ; \quad R_{a}(x, 0)= \begin{cases}1 & \text { for }-a \leq x<0 \\ 0 & \text { otherwise }\end{cases}
$$

Then, from 13, one gets

$$
P_{12}(a)=-\partial_{a}^{2} \int \mathrm{d} x R_{a}(x, t) .
$$

Figure 3 shows our numerical result for the distribution $P_{12}(a)$ of the distance between the two rightmost points in the long time limit. More details on our numerical procedure is given in appendix $\mathrm{A}$.

We see that $P_{12}(a)$ is very close to $2 e^{-2 a}$ for the values of $a$ which have a significant probability of occurring. This is of course consistent with an average distance 53 ) close to $1 / 2$. For large $a$ (events with a small probability), however, the exponential decay is faster. We now present a simple argument leading to the following prediction, which is consistent with our numerical data,

$$
P_{12}(a) \sim e^{-(1+\sqrt{2}) a} \quad \text { for large } a .
$$

In the long time limit, the right frontier of the branching Brownian motion moves at velocity $v=2$. Let us assume that a large distance $a$ between the two rightmost points is produced by the following scenario: by a rare fluctuation, the rightmost point escapes and, without branching, goes significantly ahead while the 

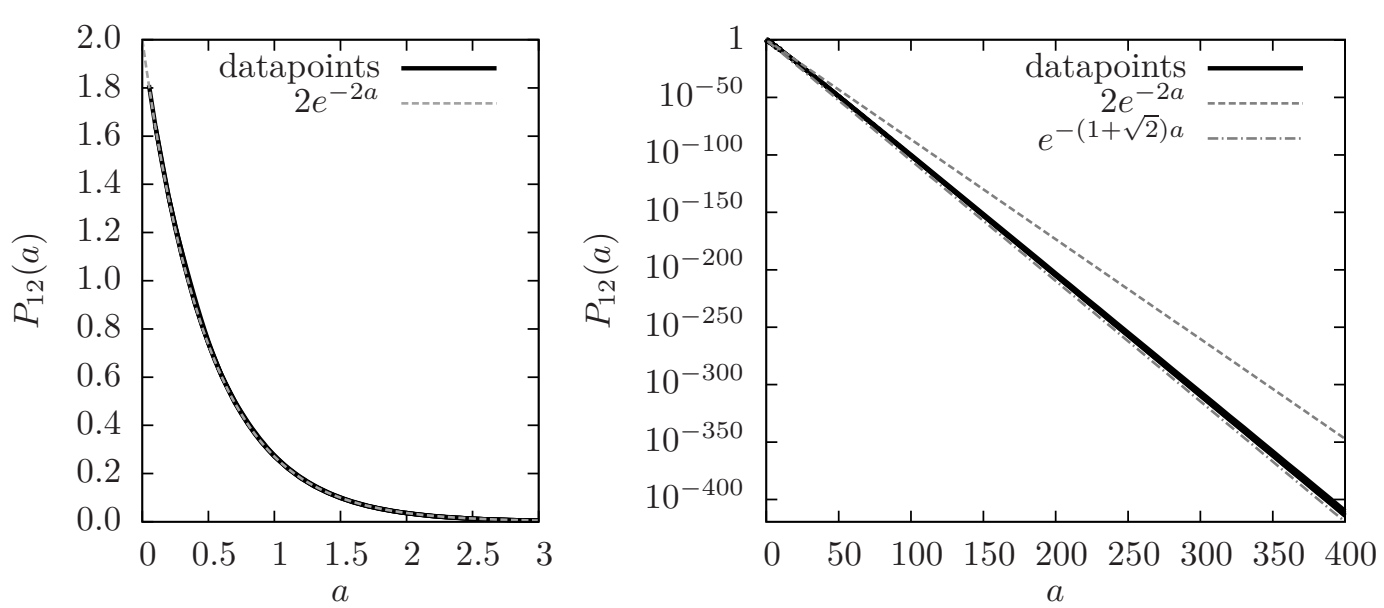

Fig. 3 The density of probability $P_{12}(a)$ of observing a distance $a$ between the two rightmost points in the $t \rightarrow \infty$ limit, as a function of $a$. For $a$ small (left part), the distribution is very close to $2 e^{-2 a}$. For larger values of $a$, one observes a faster exponential decay of order $e^{-(1+\sqrt{2}) a}$.

rest of the points go on as usual, forming a frontier moving at velocity $v=2$. Such an event leads to the distance $a$ between the two rightmost points if, during a time $\tau$, the rightmost point moves (by diffusion alone) by a distance $a+2 \tau$ without branching. The probability of such a scenario is

$$
\operatorname{Proba}\left(X_{1}-X_{2} \simeq a \text { after an escape time } \tau\right) \sim \exp \left[-\frac{(a+2 \tau)^{2}}{4 \tau}\right] \times e^{-\tau}
$$

The first term is the probability of diffusing over a distance $a+2 \tau$ during time $\tau$, and the second term is the probability of not branching. The probability to observe a large distance $a \gg 1$ is then dominated by the events with $\tau$ chosen to maximize [58), that is

$$
\tau_{\text {optimal }}=\frac{a}{2 \sqrt{2}}
$$

and this leads to 57 in good agreement with the numerical data of figure 3

There is a remarkable relation between the decay rate in (57) and the shape of the traveling wave solution of (41). Around the stable region $F=0$, the equation (41) can be linearized and one has

$$
F(z) \simeq \text { Cste } \times e^{r z} \text { for } z \rightarrow-\infty, \quad \text { with } \quad r^{2}+2 r-1=0 .
$$

We emphasize that this is a linear analysis of the stable region, which is usually uninteresting (in contrast to the unstable region which determines the velocity). The solutions for $r$ are

$$
r=-1 \pm \sqrt{2}
$$

$r=-1+\sqrt{2}$ is the correct root as it is the only positive solution and $F(-\infty)$ has to vanish. The other solution $r=-1-\sqrt{2}$ (the wrong root) coincides (up to the sign) with the decay rate of the distribution $P_{12}(a)$ for the distance $a$ between the two rightmost points 57 .

As explained in appendix B, this coincidence exists in a broad class of branching processes: each variant of the branching Brownian motion is linked to a variant of the Fisher-KPP equation, and the wrong root in the linear analysis of the stable region always gives the asymptotic decay rate of $P_{12}(a)$. 
4.3 Average density seen from the rightmost point

To obtain the average density of points at a distance $a$ on the left of the rightmost point, one needs, according to (14), to calculate $\psi_{0 \mu}(x, x-a)$ for $\mu$ close to 1 . As in section 4.2 we first remark, from the definition (7), that $\psi_{01}(x, x-a)=\operatorname{Proba}[n(x)=0]=H_{\theta}(x, t)$ is the standard Fisher-KPP front with the step initial condition. Then, writing at time $t$ that

$$
\psi_{0 \mu}(x, x-a)=H_{\theta}(x, t)-(1-\mu) \tilde{R}_{a}(x, t)+\mathscr{O}\left[(1-\mu)^{2}\right]
$$

is solution of the Fisher-KPP equation, and using the initial condition $\phi_{2}$ in [34], one gets

$$
\partial_{t} \tilde{R}_{a}=\partial_{x}^{2} \tilde{R}_{a}-\tilde{R}_{a}+2 H_{\theta} \tilde{R}_{a} ; \quad \tilde{R}_{a}(x, 0)= \begin{cases}1 & \text { for } 0 \leq x<a \\ 0 & \text { otherwise }\end{cases}
$$

It is the same equation as for $R_{a}$ in (55), but with a different initial condition. Then, from (14) one gets

$$
\rho(a)=\partial_{a}^{2} \int \mathrm{d} x \tilde{R}_{a}(x, t)
$$

One can notice the great similarity between the expressions for the average density $\rho(a)$ of points at a distance $a$ from the rightmost 6364 and the probability distribution $P_{12}(a)$ for the distance between the two rightmost points $(55 \mid 56)$ : one goes from one to the other by simple changes of signs, as in the example of a Poisson process (18).

Figure 4 presents our numerical results for $\rho(a)$ in the long time limit. We see that $\rho(a)$ increases as

$$
\rho(a) \simeq \text { Cste } \times a e^{a} \quad \text { for large } a .
$$

Note that a Poisson process with such a density would lead to asymptotic distances between points given by (52). The branching Brownian motion is however not a Poisson process as the points are correlated, at least near the tip.

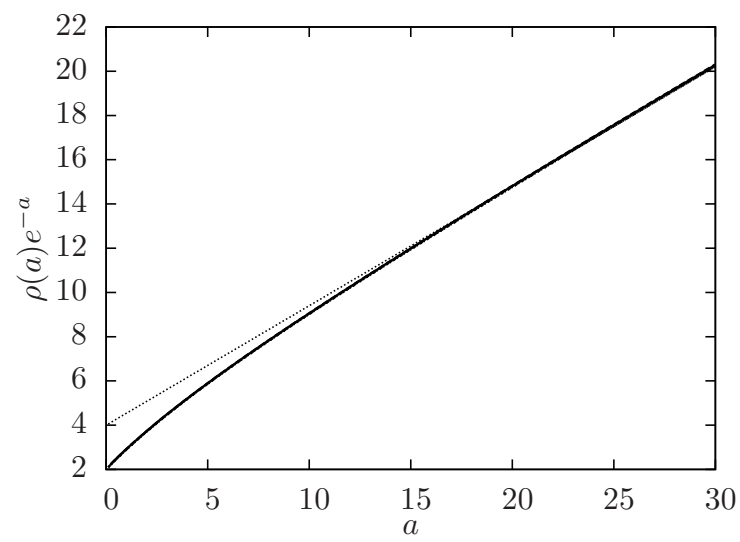

Fig. 4 The average density $\rho(a)$ of points at a distance $a$ of the rightmost in the long time limit grows like $a e^{a}$. When the data is multiplied by $e^{-a}$, as shown in the figure, the linear prefactor is clearly visible. 


\section{Generalizations to other branching processes}

All the results of sections 3 and 4 can be generalized to other branching processes on the line where points move and branch independently of the positions and of the motions of the other points. In such systems, the function $H_{\phi}(x, t)$ defined in 25 is also solution of an equation similar to the Fisher-KPP equation (27). Here are four examples:

A) The points perform Brownian motions and branch as before, but at each branching event there is a probability $p$ to branch into three points and $1-p$ to branch into two. Then $H_{\phi}(x, t)$ evolves according to

$$
\partial_{t} H_{\phi}=\partial_{x}^{2} H_{\phi}+p H_{\phi}^{3}+(1-p) H_{\phi}^{2}-H_{\phi} .
$$

B) Time is discrete with steps of duration $\delta$; at each time step, a point at position $x$ branches into two points at positions $x+\varepsilon_{1}$ and $x+\varepsilon_{2}$, where the $\varepsilon_{i}$ take independent random values distributed according to some given $\rho(\varepsilon)$. The evolution of $H_{\phi}(x, t)$ is then given by

$$
H_{\phi}(x, t+\delta)=\left[\int \mathrm{d} \varepsilon \rho(\varepsilon) H_{\phi}(x-\varepsilon, t)\right]^{2} .
$$

In this example, the positions of the points can be thought of as the possible energies of a directed polymer on a Caley tree with independent random energies $\varepsilon$ on the edges of the tree [14,27].

C) Time is continuous but space is discrete with steps 1 ; during $\mathrm{d} t$, each point at position $x$ has a probability $\mathrm{d} t$ of being removed and replaced by two points at position $x+1$. The equation satisfied by $H_{\phi}(x, t)$ is

$$
\partial_{t} H_{\phi}(x, t)=H_{\phi}(x-1, t)^{2}-H_{\phi}(x, t) .
$$

This example is relevant to the theory of binary search trees [28,24,23].

D) Time and space are discrete with steps $s$ for space and $\delta$ for time; in a given time step, a point at position $x$ has a probability $\delta$ of branching into two points at position $x$, a probability $\delta / s^{2}$ of jumping to the left, $\delta / s^{2}$ of jumping to the right, and $1-\delta-2 \delta / s^{2}$ of doing nothing. Then:

$$
H_{\phi}(x, t+\delta)=H_{\phi}(x, t)+\delta\left[\frac{H_{\phi}(x-s, t)+H_{\phi}(x+s, t)-2 H_{\phi}(x, t)}{s^{2}}-H_{\phi}(x, t)+H_{\phi}(x, t)^{2}\right],
$$

which is of course a discretized version of the original Fisher-KPP equation. 69) is actually the equation we used in our numerical simulations, see appendix A.

In all cases, these equations have $H_{\phi}=1$ as an unstable fixed point, and $H_{\phi}=0$ as a stable fixed point. For initial conditions $H_{\phi}(x, 0)=\phi(x)$ of the type 31 32 34), the function $H_{\phi}(x, t)$ develops into a traveling wave moving at a specific velocity $v^{*}$. We recall briefly the procedure to determine the asymptotic velocity $v^{*}$ of the front (which is also, through (33), the velocity of the rightmost point in the branching process). One looks for traveling wave solutions moving at velocity $v$ of the form $H_{\phi}(x, t)=F(x-v t)$ and solve the linearized equation around the unstable fixed point by writing $1-F(x) \simeq \varepsilon e^{-\gamma x}$. This leads to a relation between $\gamma$ and $v$, and the minimal value $v^{*}$ of $v$ reached at some $\gamma^{*}$ is the velocity selected by the front [31]. (We only consider here cases where the function $v(\gamma)$ has a minimum.) For our four examples

$$
\begin{array}{ll}
\text { A) } \quad v=\gamma+\frac{1+p}{\gamma} ; v^{*}=2 \sqrt{1+p}, & \text { B) } \quad v=\frac{1}{\gamma \delta} \ln \left[2 \int \mathrm{d} \varepsilon \rho(\varepsilon) e^{\gamma \varepsilon}\right] \\
\text { C) } v=\frac{2 e^{\gamma}-1}{\gamma} ; v^{*} \simeq 4.311, & \text { D) } \quad v=\frac{1}{\gamma \delta} \ln \left[1+2 \delta \frac{\cosh (\gamma s)-1}{s^{2}}+\delta\right] .
\end{array}
$$

Once the equation for $H_{\phi}$ of a particular branching process is written, one has access to all the generating functions $\psi_{\lambda}(x), \psi_{\lambda \mu}(x, x-a)$, etc., see 2779$)$, by choosing the appropriate initial conditions 
(31 34 for the front equation. The whole measure in the frame of the rightmost point is then obtained from (39) at any finite time $t$. Note that to prove the existence of a long time limit to the point measure in this frame for a specific branching process, one would need a version of Bramson's result (42) for this process which is, to our knowledge, not known in the general case.

It is natural to ask which properties of the branching Brownian motion can be extended to other branching processes. If the measure for the distances between the rightmost points has a long time limit, then the arguments of section 3.4 can be easily generalized and one can show that it is superposable. We have checked that the analytical argument [9] leading to the asymptotic expression (52) for the average distances $\left\langle d_{i, i+1}\right\rangle$ at large times can be extended in case B) for a large class of densities $\rho(\varepsilon)$ and yields

$$
\left\langle d_{i, i+1}\right\rangle \simeq \frac{1}{\gamma^{*}}\left(\frac{1}{i}-\frac{1}{i \ln i}\right) \quad \text { for large } i .
$$

We have also checked numerically on examples C) and D) that the density at a distance $a$ of the rightmost point is, as in 65,

$$
\rho(a) \simeq \text { Cste } \times a e^{\gamma^{*} a} \quad \text { for large } a .
$$

For the tail of the distribution $P_{12}(a)$ of the distance $a$ between the two rightmost points, we discussed a scenario, at the end of section 4.2 , which can be generalized (see appendix $B$ ) to calculate the exponential decay $P_{12}(a)$ for more general branching processes. This scenario, however, can only hold if points can move without branching, as in our examples A) and D); for instance, in example A), it predicts an exponential with a decay rate equal to $\sqrt{1+p}+\sqrt{2+p}$. In examples B) and C), the points branch whenever they move and the tail of $P_{12}(a)$ is in general not an exponential.

Note that special care should be taken if the points are located on a discrete lattice, as in cases C), D) and possibly B): quantities such as $P_{12}(a)$, see $(13$, become probabilities rather than densities of probability and quantities such as $\rho(a)$, see $(14)$, become average numbers rather than average densities, and all the formulas in the previous sections need to be adapted: integrals become discrete sums, derivatives become finite differences, etc. If one interprets $n(x)$ as the number of points strictly on the right of $x$, then the generating functions $\psi_{\lambda}, \psi_{\lambda \mu}, \ldots$ are still related to $H_{\phi}$ as in 30 35 with the choices 3134 for the initial condition $\phi$. Then, for instance, one can show easily that 6 becomes

$$
\sum_{x} x\left[\psi_{0}(x)-\psi_{0}(x-s)-\psi_{\lambda}(x)+\psi_{\lambda}(x-s)\right]=\sum_{i \geq 1}\left\langle d_{i, i+1}\right\rangle \lambda^{i}
$$

where $s$ is the lattice spacing. For these systems on the lattice, there are new properties that can be investigated. As an example, if $N$ is the number of points on the rightmost occupied site, then it is easy to check that

$$
\left\langle\mu^{N}\right\rangle=1+\sum_{x}\left[\psi_{0 \mu}(x, x-s)-H_{\theta}(x, t)\right] .
$$

The whole distribution of $N$ can then be determined by numerical integration. In the case of our example $\mathrm{C}$ ), the number $N$ corresponds to the number of leaves at the deepest level in a binary search tree [28] and we found numerically that, at large times, $\operatorname{Proba}(N=2) \simeq 0.50, \operatorname{Proba}(N=4) \simeq 0.23$, $\operatorname{Proba}(N=6) \simeq 0.11, \operatorname{Proba}(N=8) \simeq 0.06$, etc.

\section{Large time measure in the frame of Lalley and Sellke}

We recall from the results of section 3.1, see (40) and (33), that the distribution of the rightmost point is given in the long time limit by

$$
\lim _{t \rightarrow \infty} \operatorname{Proba}\left[X_{1}(t)<m_{t}+x\right]=F(x),
$$


where $m_{t}$ is the average position of the rightmost point and is asymptotically given by (42) and where $F$ is defined in (41). The property (75) is however non-ergodic, as shown by Lalley and Sellke [22] in the sense that for a single realization of the branching Brownian motion

$$
\text { fraction of the time that } \left.X_{1}(t)<m_{t}+x\right] \neq F(x) \text {. }
$$

In fact, the left hand side of $(76)$ is not self-averaging and depends on the realization. This is illustrated in figure 5 for the realization on the left, there were few branching events at early times and the first points wandered to the left, leading at larger times to an asymmetric picture. For the realization on the right of figure 5, there were many branching events early. For the right realization, the rightmost point is almost always on the right of $m_{t}$ while it is almost always on the left of $m_{t}$ in the left realization.
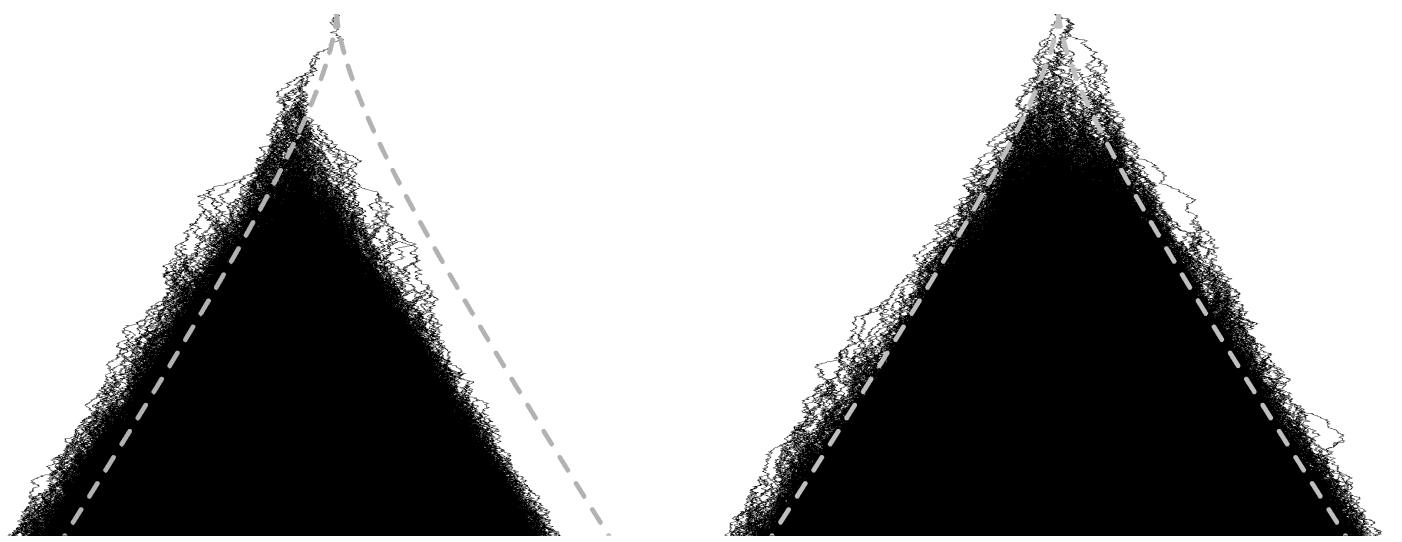

Fig. 5 Two realizations of the branching Brownian motion up to time $t=20$. The horizontal direction represents space, and time increases downwards. The dotted gray lines are $m_{t}$ and $-m_{t}$, the average positions of the rightmost and leftmost points, as measured from equation 36 .

Visually, these strong memory effects of the early stages of the branching Brownian motion do not seem to decay with time, and it looks like the fluctuating right frontier of the system settles at some random fixed distance $C$ from $m_{t}$ :

$$
X_{1}(t)=m_{t}+C+\eta_{1}(t) \quad \text { for large } t,
$$

where $C$ would depend on the realization but not the time, and where $\eta_{1}(t)$ would be a time-dependent random number centered around zero. A natural question is whether it is possible to define $C$ for each realization in such a way that the distribution of $\eta_{1}(t)$ becomes in the long time limit independent of $C$ and $t$, the idea being that the branching Brownian motion at long times seen from $m_{t}+C$ would "look the same" for any realization, whatever is the value of $C$.

A related question was addressed by Lalley and Sellke [22] in the following way: for each realization of the branching Brownian motion, define $Z$ as

$$
Z=\lim _{t \rightarrow \infty} Z_{t} \quad \text { where } Z_{t}=\sum_{i}\left[2 t-X_{i}(t)\right] e^{X_{i}(t)-2 t} .
$$

(The sum is over all the points $X_{i}(t)$ present at time $t$.) As shown in [22], $Z_{t}$ has a limit $Z$ for almost every realization; that limit is finite and positive. Lalley and Sellke prove then a limit theorem for the frontier of the branching Brownian motion which we interpret as follows:

$$
\lim _{t \rightarrow \infty} \operatorname{Proba}\left(X_{1}(t)<m_{t}+x \mid Z\right)=\exp \left(-A Z e^{-x}\right)=\exp \left(-e^{-[x-\ln (A Z)]}\right),
$$


where $A$ is a constant related to the large $x$ behavior of $F(x)$, see 101 . In words, if one considers only the realizations of the branching Brownian motion with a given value of $Z$, then the large time distribution of the rightmost point is given by a Gumble located around $m_{t}+\ln (A Z)$. To make the link with (77),

$$
C=\ln (A Z), \quad \operatorname{Proba}\left(\eta_{1}<x\right)=\exp \left(-e^{-x}\right) .
$$

In appendix C, we present the precise theorem stated by Lalley and Sellke and we argue that (79) should be equivalent to their result.

A natural extension to Lalley's and Sellke's result is to write for all the points $i$

$$
X_{i}(t)=m_{t}+\ln (A Z)+\eta_{i}(t),
$$

as in (77), and ask whether the joint distribution of $\eta_{1}(t), \eta_{2}(t), \eta_{3}(t), \ldots$ reaches a long time limit which is independent of the value of $Z$. We show in appendix C that Lalley's and Sellke's result can be extended to all the generating functions $H_{\phi}$. Our interpretation of this extension is

$$
\lim _{t \rightarrow \infty}\left\langle\prod_{i} \phi\left(m_{t}+x-X_{i}(t)\right) \mid Z\right\rangle=\exp \left(-A Z e^{-x-f[\phi]}\right)=\exp \left(-e^{-[x-\ln (A Z)+f[\phi]]}\right),
$$

where the delay function $f[\phi]$ is the same as in 445 . By choosing $\phi=\theta$ (the step function), 82 reduces to 79 . By choosing $\phi$ as in (31), one sees from (82) that the distribution of points at the right of the branching Brownian motion conditioned by $Z$ reaches a long time limit where $Z$ only appears through the global shift $\ln (A Z)$. This means that at large times, the distribution of the rightmost points in a branching Brownian motion has a well defined measure independent of $Z$ located around $m_{t}+\ln (A Z)$.

As an example, if one chooses the function $\phi_{1}$ defined by (34), one can easily show from (82) and (51) that, in the $m_{t}+\ln (A Z)$ frame, the average density of points at any position is infinite in the long time limit.

\subsection{Superposability property}

If one considers two branching Brownian motions $a$ and $b$ starting at arbitrary positions, then the points in $a$ at large time will be characterized by a random value $Z^{(a)}$ and a realization of the point measure described by (82); idem for the points in $b$. If one considers the union of these two branching Brownian motions, one gets from 82 )

$$
\lim _{t \rightarrow \infty}\left\langle\prod_{i} \phi\left(m_{t}+x-X_{i}(t)\right) \mid Z^{(a)}, Z^{(b)}\right\rangle=\exp \left(-A Z e^{-x-f[\phi]}\right)=\exp \left(-e^{-[x-\ln (A Z)+f[\phi]]}\right),
$$

with $Z=Z^{(a)}+Z^{(b)}$. This means that the point measure reached in the long time limit in the $m_{t}+\ln (A Z)$ frame is the same whether one started initially with one, two or, by extension, any finite number of initial points at arbitrary positions on the line. What does depend on the initial number of points is only the law of the random number $Z$, not the positions around $m_{t}+\ln (A Z)$. This is to be related to the discussion in section 3.4, where we showed that, in the long time limit, the measure seen from $m_{t}$ depends on the initial number of points while the measure seen from $X_{1}(t)$ does not.

Furthermore, the large time measure of the points in the $m_{t}+\ln (A Z)$ frame has the following property:

Starting with two realizations $\left\{\eta_{i}^{(a)}\right\}$ and $\left\{\eta_{i}^{(b)}\right\}$, then for any pair of real numbers $\alpha$ and $\beta$, the ensemble of points $\left\{\eta_{i}^{(a)}+\alpha\right\} \cup\left\{\eta_{i}^{(b)}+\beta\right\}$ is another realization of the same measure shifted by $\ln \left(e^{\alpha}+e^{\beta}\right)$.

(Think of $\left\{\eta_{i}^{(a)}\right\}$ as the offspring of $a$ in the $m_{t}+\ln \left[A Z^{(a)}\right]$ frame and of $\alpha$ as $\ln Z^{(a)}$; idem for $b$. The shifts $\alpha$ and $\beta$ are arbitrary because $Z^{(a)}$ and $Z^{(b)}$ are unbounded independent random numbers.) 
The property (84) of the point measure in the $m_{t}+\ln (A Z)$ frame is a stronger version of the superposability property discussed in section 3.4 clearly, it implies that the distribution of distances between the rightmost points is invariant by superposition, but it gives more information on the measure as it encompasses the position of the rightmost point. In particular, one can check that, in any such measure, the rightmost point is Gumble distributed.

The simplest point measure with the property (84) is the Poisson process with an exponential density $K e^{-x}$, for an arbitrary $K$. Furthermore, all the decorated measures of section 2.3.3 when $v_{1}$ is a Poisson process with an exponential density $K e^{-x}$ are also superposable measures. A natural question is then: can any superposable point measure be constructed as a decorated exponential Poisson process for a well chosen decoration measure ? A more specific question would be: is the limiting point measure of the branching Brownian motion in the $m_{t}+\ln (A Z)$ frame can be constructed as a decorated exponential Poisson process?

We conjecture that the answer to the last question is yes. Indeed, it is known [14,3] that if one considers two points chosen at random among the rightmost points in a branching Brownian motion at a large time $t$, then the time one needs to go back to find the most recent common ancestor of those two points is either very short (of order 1) or very long (of the order of the age $t$ of the system). This means that one can group the rightmost points into families where two points belong to the same family if the branching event that generated them occurred recently. The branching Brownian motion could then be a decorated exponential Poisson process, where the rightmost points of all the families are Poisson distributed with an exponential density [3] and where the members of a given family form the decoration. This interpretation helps to understand a question raised by our previous work [9]: the distances between points in the branching Brownian motion are given by (52 53), but if one considers only the rightmost point of each family, then the distances are given by (21) with $\alpha=1$, as in the GREM or in the Ruelle cascade, see section 2.3 .4

\section{Conclusion}

In the present work, we have shown that all the statistical properties of the rightmost points in a branching Brownian motion can be obtained by solving a front equation with a well-chosen initial condition. The distribution of the positions of the rightmost points seen in the frame $m_{t}$ (the average position of the rightmost) has a long time limit. The properties of the limiting distribution can be expressed as the long time delays of the traveling wave solution of the Fisher-KPP equation when one varies the initial condition. This limiting distribution is however modified if one considers the union of several branching Brownian motions.

If one considers, however, only the distances between the points, for example if one looks at the distribution of all the positions of the rightmost points seen in the frame of the rightmost one, one obtains at large times another limiting distribution which does not depend on the initial positions of the branching Brownian motions (as long as there are finitely many of them). We called this property superposability.

In section 4 we have measured a few properties of this limiting distribution, and in section 5 we explained how our results can be extended to more general branching random walks. Lastly, in section 6 . we argued that in the Lalley and Sellke frame, the branching Brownian motion satisfies a stronger version of the superposability property, see 84 .

In the future, it would be interesting to characterize more precisely the limiting measure of the branching Brownian motion and of the branching random walks to see whether some universal properties emerge. For example, we believe that the average density seen from the rightmost point should always grow as in (72). It would also be interesting to check whether, in Lalley's and Sellke's frame, the limiting distribution is indeed a decorated exponential, and to determine the properties of the decorating measure. 
The question of ergodicity, raised at the beginning of section 6 , is also an interesting open question.

Acknowledgements We would like to thank Julien Berestycki and Simon Harris for interesting discussions.

\section{A Numerical simulations}

We performed the numerical simulations by discretizing the branching Brownian motion in space (with a grid length $\delta$ ) and time (with steps $s$ ) as in the example D) of section 5 The corresponding front equation is given by 69, which is of course the most straightforward discretization of the Fisher-KPP equation [27]. The solutions to the discrete equation 69] converge to the solutions of the Fisher-KPP equation 27, if $s \rightarrow 0$ with $\delta / s^{2}$ held constant and small enough.

We used three sets of values for $s$ and $\delta$ and computed the exact asymptotic velocity $v^{*}$ of the front and the decay rate $\gamma^{*}$ of the asymptotic shape $F(x)$ by minimizing numerically the function $v(\gamma)$ given in $70 \mathrm{P})$. The decay rate $\beta$ for the probability of observing a distance $a$ between the two rightmost points, see equation 57, was computed using the recipe given at the end of section 4.2 and explained in appendix B All these values are presented in table 1

\begin{tabular}{|cl|ccc|}
\hline$s$ & \multicolumn{1}{c|}{$\delta$} & $v^{*}$ & \multicolumn{1}{c|}{$\gamma^{*}$} & $\beta$ \\
\hline 0.25 & $s^{2} / 5=0.0125$ & 1.980480133 & 1.004581693 & 2.387337826 \\
0.10 & $s^{2} / 5=0.002$ & 1.996840367 & 1.000747277 & 2.409772891 \\
0.05 & $s^{2} / 6 \simeq 0.000416667$ & 1.999375296 & 1.000104046 & 2.412897517 \\
\hline \multicolumn{2}{|c|}{ Fisher-KPP } & 2 & 1 & $\sqrt{2}+1=2.414213562$ \\
\hline
\end{tabular}

Table 1 Values of $v^{*}, \gamma^{*}$ and $\beta$ for our discretized branching processes, compared to the values in the Fisher-KPP case.

The simulations were made on a finite but large domain centered around the position of the front; typically it extended to about a distance 1000 ahead and behind the center of the front (respectively 4000, 10000 or 20000 lattice sites depending on $s$ ). The values at $x= \pm \infty$ were exactly computed and used for the boundaries of the domain. Whenever the front moved by more than one unit space lattice, the whole data set was recentered. The simulations were performed up to large times of order 10000 ( 0.8 to 24 millions of time steps) and the data was extrapolated to obtain a value at $t=\infty$. To do this extrapolation, we used a more precise asymptotic expansion of the position of the front than [42): according to [15],

$$
m_{t}=v^{*} t-\frac{3}{2 \gamma^{*}} \ln t+\text { Constant }+\frac{a_{1 / 2}}{t^{1 / 2}}+\frac{a_{1}}{t}+\frac{a_{3 / 2}}{t^{3 / 2}}+\cdots,
$$

where the number $a_{1 / 2}$ does not depend on the initial condition. As we measure the delay $m_{t}-m_{t}^{(\phi)}$, many terms cancel and one gets

$$
m_{t}-m_{t}^{(\phi)}=f[\phi]+\frac{\delta a_{1}}{t}+\frac{\delta a_{3 / 2}}{t^{3 / 2}}+\cdots .
$$

All the quantities we measure are derivatives of $f[\phi]$, see $[47$, and have therefore the same large time expansion as 86 . Thus, we extrapolated our numerical data to the large time limit by fitting it with the function $A+B / t+C / t^{3 / 2}$ for times larger than (typically) 5000 , see figure 6 and by using $A$ as the end result.

On figure 3 the three data points were presented together; on figure 4 we have drawn together for each data set the function $\rho\left(X_{1}-a\right) e^{-\gamma^{*} a}$ using in each case the value of $\gamma^{*}$ of table 1 In both cases, the superposition was nearly perfect, and so we expect that on the scales of the figure, the curves would not change noticeably for smaller values of $s$ and $\delta$.

\section{B Distribution of the distance between the two rightmost points}

In this appendix we generalize, to any branching random walk, the argument leading to the asymptotic decay [57] of the distribution of the distance between the two rightmost points in the branching Brownian motion.

We consider a generic branching random walk in discrete space (with spacing $s$ ) and time (with intervals $\delta$ ) defined by the following family of functions

$$
p_{n}\left(r_{1}, \ldots, r_{n}\right)=\left(\begin{array}{l}
\text { The probability that a point at position } x \text { branches during a time } \\
\text { step into } n \text { points located at positions } x+r_{1}, \ldots, x+r_{n} .
\end{array}\right) .
$$




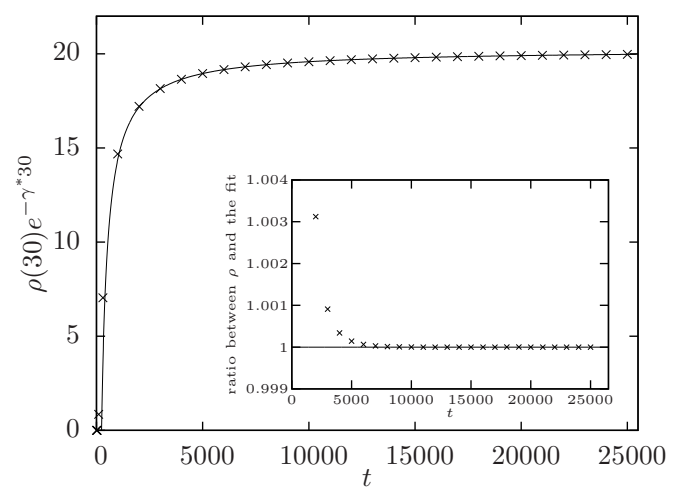

Fig. 6 Average density at a distance 30 from the rightmost point as a function of time (symbols), fitted for $t \geq 5000$ by the function $A+B / t+C / t^{3 / 2}$ (line). The inset shows the quality of the fit by displaying the ratio between the data points and the fitting function.

We assume that $p_{0}=0$, so that there is no extinction. Then $p_{1}(r)$ can be thought as the probability that the point does not branch but moves by a distance $r$. The continuous time and/or space cases can be obtained as suitable $s \rightarrow 0$ and/or $\delta \rightarrow 0$ limits.

Let $\exp [\operatorname{tg}(\beta)]$ be the generating function of the displacement during time $t$ of one point conditioned on the fact that this point does not branch:

$$
e^{t g(\beta)}=\sum_{r} e^{\beta r} \operatorname{Proba}(\text { the point moves a distance } r \text { without branching during time } t \text { ). }
$$

As the time steps are independent, the function $g(\beta)$ can be computed during the time interval $\delta$ which gives

$$
e^{\delta g(\beta)}=\sum_{r} p_{1}(r) e^{\beta r}
$$

Note that $g(0)<0$ as soon as the branching probability is non-zero. We want now to evaluate the probability that a point moves a distance $r$, without branching, during time $t$. For large $t$, it takes the form

$$
\text { Proba(the point moves a distance } r \text { without branching during time } t) \sim \exp \left[t f\left(\frac{r}{t}\right)\right],
$$

where $f(c)$ is a large deviation function. Using 88 , one finds that $f(c)$ and $g(\beta)$ are related by a Legendre transform

$$
\left\{\begin{array}{l}
\beta=-f^{\prime}(c) \\
g(\beta)=f(c)+\beta c .
\end{array}\right.
$$

Now, assuming as in section 4.2 that the events which contribute most to a large distance $a$ between the two rightmost points are those where the rightmost point moves, without branching, a distance $a$ ahead of the frontier of the branching Brownian motion, one gets

$$
P_{12}(a) \sim \max _{\tau}\left\{\exp \left[\tau f\left(\frac{a+v^{*} \tau}{\tau}\right)\right]\right\},
$$

where $v^{*}$ is the velocity of the front. For large $a$, the optimal $\tau$ is also large and it satisfies, by derivation,

$$
f\left(\frac{a}{\tau}+v^{*}\right)-\frac{a}{\tau} f^{\prime}\left(\frac{a}{\tau}+v^{*}\right)=0 .
$$

Let $c=a / \tau+v^{*}$. Using (91), equation 93 becomes

$$
g(\beta)=\beta v^{*} .
$$

Remarkably, this equation does not depend on $a$. Replacing into $[92]$ gives

$$
P_{12}(a) \sim e^{-\beta a} .
$$

The asymptotic decay rate of the probability distribution function of the distance between the two rightmost points is therefore simply the positive solution $\beta$ of 94 with $g(\beta)$ given by 89 . In the branching Brownian motion, $g(\beta)=\beta^{2}-1, v^{*}=2$, so that (94) gives indeed $\beta=1+\sqrt{2}$.

As can be checked easily from 89 , the function $g(\beta)$ is convex. Therefore, as $g(0)<0$, equation 94 has at most one positive solution and at most one negative solution. The positive solution is the relevant one here. 
We are now going to show that the negative solution of 94 , if it exists, gives the asymptotic shape of the associated traveling wave. We write the front equation associated to the branching point process 87 . Using the same method as in section 3 we find

$$
H_{\phi}(x, t+\delta)=\sum_{n \geq 1} \sum_{r_{1}, \ldots, r_{n}} p_{n}\left(r_{1}, \ldots, r_{n}\right) \prod_{i=1}^{n} H_{\phi}\left(x-r_{i}\right)
$$

We look at the shape $F$ of the traveling wave solution for a step initial condition, which moves asymptotically at the velocity $v^{*}$ :

$$
H_{\phi}(x, t)=F\left(x-v^{*} t\right) .
$$

Using [96, we see that in the stable region $F(x) \ll 1$ one has

$$
F\left(x-v^{*} \delta\right)=\sum_{r} p_{1}(r) F(x-r)+\mathscr{O}\left(F^{2}\right)
$$

We look for an exponential solution to this linearized equation: $F(x) \simeq e^{\lambda x}$ with $\lambda>0$, as $F(-\infty)=0$. (Note that a periodic modulation of this exponential could occur as $r$ takes only discrete values.) Inserting into [98] and using [89], one finds that the equation for $\lambda$ is

$$
g(-\lambda)=-\lambda v^{*}
$$

which is the same equation as 94 for $\lambda=-\beta$.

To summarize, a positive solution to 94 gives the exponential decay rate of the probability distribution of the distance between the two rightmost points, see 95, while a negative solution gives the coefficient $-\lambda$ governing the shape of the front $F(x)$ in the stable region $F(x) \ll 1$.

\section{Lalley's and Sellke's result}

Lalley's and Sellke's theorem [22] is

$$
\lim _{s \rightarrow \infty} \lim _{t \rightarrow \infty} \operatorname{Proba}\left(X_{1}(t)<m_{t}+x \mid\left\{X_{i}(s)\right\}\right)=\exp \left(-A Z e^{-x}\right),
$$

where $A$ is the constant appearing [8] in the large $x$ expansion of the function $F(x)$ defined in 41

$$
F(x) \simeq 1-(A x+B) e^{-x} \quad \text { for large } x,
$$

and $Z$ is defined in 78 . In words, given the positions $\left\{X_{i}(s)\right\}$ at time $s$, there is a $t \rightarrow \infty$ limit to the probability that the rightmost is on the left of $m_{t}+x$ which depends, obviously, on the $\left\{X_{i}(s)\right\}$ and is as such a random variable. As $s$ goes to infinity, this random variable converges almost surely to the Gumble distribution around $\ln (A Z)$.

This result can be extended into the following: for any suitable function $\phi$ (see section 3.2 , , one has

$$
\lim _{s \rightarrow \infty} \lim _{t \rightarrow \infty}\left\langle\prod_{i} \phi\left[m_{t}+x-X_{i}(t)\right] \mid\left\{X_{i}(s)\right\}\right\rangle=\exp \left(-A Z e^{-x-f[\phi]}\right),
$$

where $f[\phi]$ is the delay function 45 . For $\phi=\theta$, 102 reduces to 100 .

We first give an outline of Lalley's and Sellke's proof applied to the case 102 . Given the positions $X_{i}(s)$ of the points at time $s$, the system as time $t>s$ can be seen as a collection of independent branching Brownian motions at time $t-s$ starting from the $X_{i}(s)$. Therefore

$$
\left\langle\prod_{i} \phi\left[x-X_{i}(t)\right] \mid\left\{X_{i}(s)\right\}\right\rangle=\prod_{i} H_{\phi}\left(x-X_{i}(s), t-s\right),
$$

where the product in the right hand side is made on all the points present at time $s$.

We replace $x$ by $m_{t}+x$, to center around the position of the front, and suppose $t$ large. It is easy to see from Bramson's formula 42) that $m_{t}=m_{t-s}+2 s+o(1)$ as $t$ becomes large, so that

$$
\left\langle\prod_{i} \phi\left[m_{t}+x-X_{i}(t)\right] \mid\left\{X_{i}(s)\right\}\right\rangle=\prod_{i} H_{\phi}\left(m_{t-s}+2 s+x-X_{i}(s)+o(1), t-s\right),
$$

and, using 45,

$$
\lim _{t \rightarrow \infty}\left\langle\prod_{i} \phi\left[m_{t}+x-X_{i}(t)\right] \mid\left\{X_{i}(s)\right\}\right\rangle=\prod_{i} F\left(2 s+x-X_{i}(s)+f[\phi]\right) .
$$

We now take $s$ large. Of all the points present at time $s$, the rightmost is around $2 s-\frac{3}{2} \log s$, see 42]. Therefore, $2 s-X_{i}(s)$ diverges for all $i$. Using [101,

$$
\lim _{t \rightarrow \infty}\left\langle\prod_{i} \phi\left[m_{t}+x-X_{i}(t)\right] \mid\left\{X_{i}(s)\right\}\right\rangle \simeq \exp \left(-\sum_{i}\left[A\left(2 s+x-X_{i}(s)+f[\phi]\right)+B\right] e^{-2 s-x+X_{i}(s)-f[\phi]}\right) .
$$


Following Lalley and Sellke, we introduce the quantities

$$
Y_{s}=\sum_{i} e^{-2 s+X_{i}(s)}, \quad Z_{s}=\sum_{i}\left[2 s-X_{i}(s)\right] e^{-2 s+X_{i}(s)},
$$

see (78), so that

$$
\lim _{t \rightarrow \infty}\left\langle\prod_{i} \phi\left[m_{t}+x-X_{i}(t)\right] \mid\left\{X_{i}(s)\right\}\right\rangle \simeq \exp \left(-\left[A Z_{s}+(A x+A f[\phi]+B) Y_{s}\right] e^{-x-f[\phi]}\right) .
$$

Finally, the most technical part of Lalley's and Sellke's proof is that $Y_{s}$ and $Z_{s}$ are martingales converging when $s \rightarrow \infty$ to lim $\rightarrow \rightarrow \infty Y_{s}=$ 0 and $\lim _{s \rightarrow \infty} Z_{s}=Z>0$ respectively, which leads to 102 . We do not reproduce this part of the proof here as it does not concern our extension with the function $\phi$ and it works in 102 exactly as in 1100 .

In (102), the average is made on all the realizations with a given set $\left\{X_{i}(s)\right\}$ of points at a large time $s$ but the only relevant quantity appearing in the generating function 108 is $Z_{s}$. One would obviously have reached the same result if one had conditioned by $Z_{s}$ instead of by the $\left\{X_{i}(s)\right\}$. Furthermore, as $Z_{s}$ converges quickly to $Z$, as illustrated on figure 5 we argue that conditioning by $Z_{s}$ at a large time $s$ or directly conditioning by $Z$ should be equivalent, hence $79[82$.

\section{References}

1. Aizenman, M., Sims, R., Starr, S.L.: Mean-field spin glass models from the cavity-ROSt perspective. Contemporary Math. Series 437, 1-30 (2007)

2. Arguin, L.P.: Spin glass computations and Ruelle's probability cascades. J. Stat. Phys. 126, 951-976 (2007)

3. Arguin, L.P., Bovier, A., Kistler, N.: The genealogy of extremal particles of branching brownian motion. arXiv:1008.4386 [math.PR] (2010)

4. Bolthausen, E., Sznitman, A.S.: On Ruelle's probability cascades and an abstract cavity method. Comm. Math. Phys. 197, 247-276 (1998)

5. Bouchaud, J.P., Mézard, M.: Universality classes for extreme-value statistics. J. Phys. A 30, 7997-8015 (1997)

6. Bovier, A., Kurkova, I.: A tomography of the GREM: Beyond the REM conjecture. Comm. Math. Phys. 263, 535-552 (2006)

7. Bovier, A., Kurkova, I.: Local energy statistics in spin glasses. J. Stat. Phys. 126, 933-949 (2007)

8. Bramson, M.D.: Convergence of solutions of the Kolmogorov equation to traveling waves. Mem. Am. Math. Soc. 44(285) (1983)

9. Brunet, É., Derrida, B.: Statistics at the tip of a branching random walk and the delay of traveling waves. Europhys. Lett. 87, 60010 (2009)

10. Burkhardt, T.W., Györgyi, G., Moloney, N.R., Racz, Z.: Extreme statistics for time series: Distribution of the maximum relative to the initial value. Phys. Rev. E 76, 041119 (2007)

11. Dean, D.S., Majumdar, S.N.: Extreme-value statistics of hierarchically correlated variables deviation from gumbel statistics and anomalous persistence. Phys. Rev. E 64, 046121 (2001)

12. Derrida, B.: Random-energy model: An exactly solvable model of disordered systems. Phys. Rev. B 24, 2613-2626 (1981)

13. Derrida, B.: A generalization of the random energy model which includes correlations between energies. J. Phys. Lett. 46, L401-L407 (1985)

14. Derrida, B., Spohn, H.: Polymers on disordered trees, spin glasses and traveling waves. J. Stat. Phys. 51((5/6)), 817-840 (1988)

15. Ebert, U., van Saarloos, W.: Front propagation into unstable states: Universal algebraic convergence towards uniformly translating pulled fronts. Physica D 146, 1-99 (2000)

16. Fisher, R.A.: The wave of advance of advantageous genes. Annals of Eugenics 7, 355-369 (1937)

17. Golding, I., Kozlovsky, Y., Cohen, I., Ben-Jacob, E.: Studies of bacterial branching growth using reaction-diffusion models for colonial development. Physica A 260, 510-554 (1998)

18. Györgyi, G., Moloney, N.R., Ozogany, K., Racz, Z.: Finite-size scaling in extreme statistics. Phys. Rev. Lett. 100, 210601 (2008)

19. Igloi, F., Monthus, C.: Strong disorder RG approach of random systems. Phys. Rep. 412, $277-431$ (2005)

20. Kessler, D.A., Levine, H., Ridgway, D., Tsimring, L.: Evolution on a smooth landscape. J. Stat. Phys. 87(3/4), 519-544 (1997)

21. Kolmogorov, A., Petrovsky, I., Piscounov, N.: Étude de l'équation de la diffusion avec croissance de la quantité de matière et son application à un problème biologique. Bull. Univ. État Moscou, A 1(6), 1-25 (1937)

22. Lalley, S.P., Sellke, T.: A conditional limit theorem for the frontier of a branching Brownian motion. Ann. Prob. 15, 1052-1061 (1987)

23. Majumdar, S.N., Dean, D.S., Krapivsky, P.L.: Understanding search trees via statistical physics. Pramana J. Phys. 64(6) (2005)

24. Majumdar, S.N., Krapivsky, P.L.: Extreme value statistics and traveling fronts: Application to computer science. Phys. Rev. E 65, 036127 (2002)

25. McKean, H.P.: Applications of brownian motion to the equation of Kolmogorov-Petrovski-Piscounov. Comm. Pure Appl. Math. 28, 323-331 (1975)

26. Mézard, M., Parisi, G., Sourlas, N., Toulouse, G., Virasoro, M.A.: Replica symmetry-breaking and the nature of the spin-glass phase. Journal de Physique 45, 843-854 (1984) 
27. Monthus, C., Garel, T.: On the critical weight statistics of the random energy model and of the directed polymer on the Cayley tree. Phys. Rev. E 75(5), 051119 (2007)

28. Roberts, M.I.: Almost sure asymptotics for the random binary search tree. arXiv:1002.3896 [math.PR] (2010)

29. Ruelle, D.: A mathematical reformulation of Derrida's REM and GREM. Comm. Math. Phys 108, 225-239 (1987)

30. Ruzmaikina, A., Aizenman, M.: Characterization of invariant measures at the leading edge for competing particle systems. Ann. Prob. 33, 82-113 (2005)

31. van Saarloos, W.: Front propagation into unstable states. Phys. Rep. 386(2-6), 29-222 (2003)

32. Sabhapandit, S., Majumdar, S.N.: Density of near-extreme events. Phys. Rev. Lett. 98, 140201 (2007)

33. Tracy, C.A., Widom, H.: Level-spacing distributions and the Airy kernel. Comm. Math. Phys. 159, 151-175 (1994) 